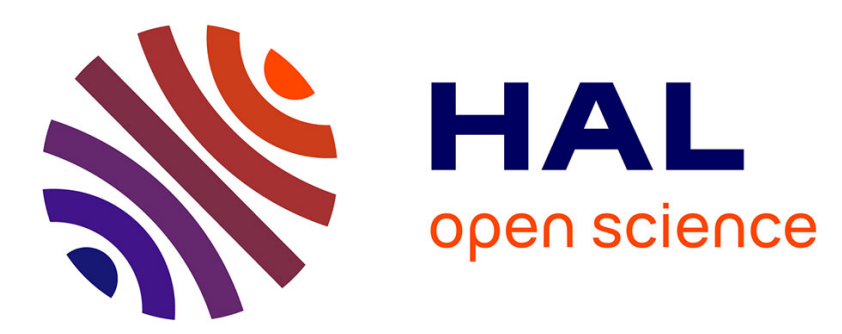

\title{
Modelling of Nonstationary Heat Conduction Problems in Micro-periodic Composites
}

\author{
Czeslaw Wozniak, Zbigniev F. Baczynski, Margaret Wozniak
}

\section{To cite this version:}

Czeslaw Wozniak, Zbigniev F. Baczynski, Margaret Wozniak. Modelling of Nonstationary Heat Conduction Problems in Micro-periodic Composites. Journal of Applied Mathematics and Mechanics / Zeitschrift für Angewandte Mathematik und Mechanik, 1996, 76 (4), pp.223-229. hal-00944365

\section{HAL Id: hal-00944365 https://hal.science/hal-00944365}

Submitted on 18 Feb 2014

HAL is a multi-disciplinary open access archive for the deposit and dissemination of scientific research documents, whether they are published or not. The documents may come from teaching and research institutions in France or abroad, or from public or private research centers.
L'archive ouverte pluridisciplinaire HAL, est destinée au dépôt et à la diffusion de documents scientifiques de niveau recherche, publiés ou non, émanant des établissements d'enseignement et de recherche français ou étrangers, des laboratoires publics ou privés. 
WOŹNIAK, C.; BaCZYŃSKI, Z. F.; WoŹNIAK, M.

\section{Modelling of Nonstationary Heat Conduction Problems in Micro-periodic Composites}

In diesem Beitrag wird ein Modell des Wämeleiters mit mikroperiodischer Struktur formuliert. Dieses angebotene Modell beschreibt den Einfluß der Struktur im zeitabhängigen Wärmeleitungsprozeß. Es wurde bewiesen, daß dieser Einfluß in allen nichtstationären Problemen eine bedeutende Rolle spielt.

In this contribution an averaged refined model of a rigid heat conductor with micro-periodic structure is formulated. The proposed model describes the effect of a microstructure length dimension on the time-dependent heat transfer processes. It is shown that this effect plays a crucial role in the analysis of nonstationary problems and hence the known effectite modulus theories of heat conduction (where the microstructure is scaled down), have the range of applicability restricted to stead!-state problems.

$\operatorname{MSC}(1991): 73 B 30,73 C 60,73 \mathrm{~K} 20,73599$

\section{Introduction}

The subject of this paper is a heat conduction in a rigid conductor, made of a micro-periodic composite material (cf. Fig. 1). Due to the highly oscillating character of thermal properties of a composite, suffering the jump discontinuities across the interfaces between constituents, the direct description of heat transfer problems cannot be used as a basis for analytical and/or numerical solutions to engineering problems. That is why different homogenized models of the heat conduction in periodic composite materials are formulated. These models are obtained, as a rule, by the asymptotic procedures where the microstructure is scaled down, cf: BeNSOUSSAN et al. [1], BAKHVALOV and PANASENKo [2], MATYSIAK and WoźNiAK [3], etc., leading to what are called "the effective modulus theories". In heat conduction problems the constant effective modulae describe in an averaged form the highly oscillating thermal properties of a medium (e.g. thermal conductivity), which in the direct description of a problem are described by the micro-periodic functions. However, the known effective modulus theories, obtained by the scaling microstructure down, do not take into account the microstructure
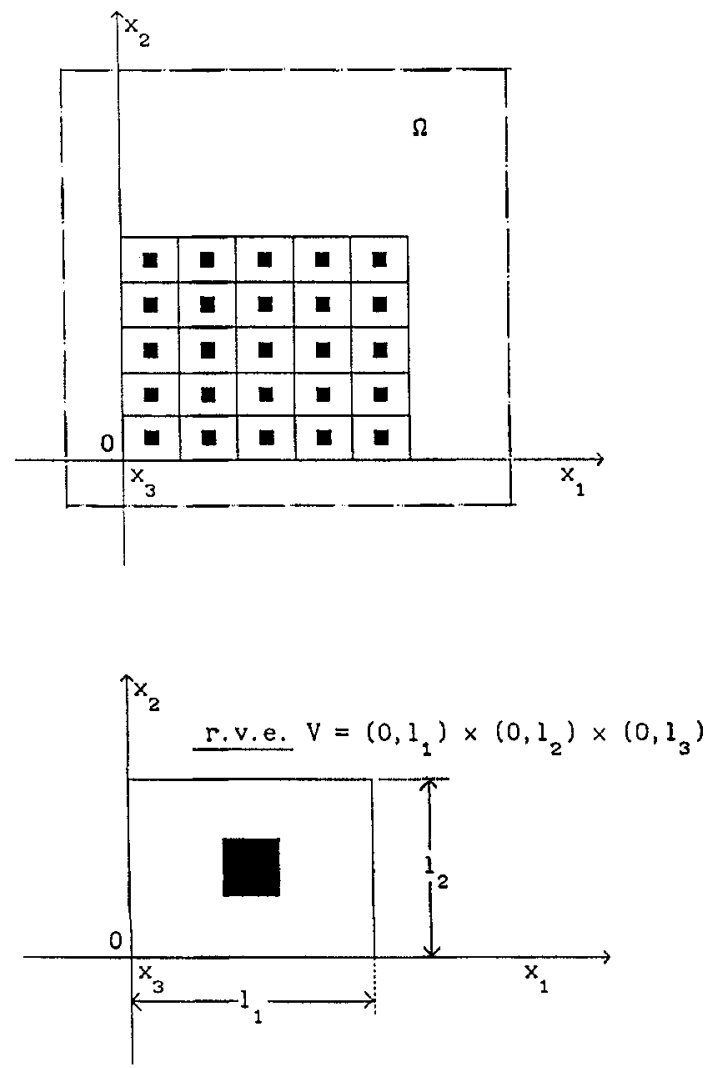

Fig. 1. Scheme of a composite body and its representative volume element 
length-scale effects on the heat transfer in composite materials. This fact stands for the motivation of the presented research, and its thesis is that in nonstationary problems length scale effects play a crucial role related both to the quantitative and qualitative character of a thermal conductivity.

The main aim of the research is to propose a certain new refined averaged model of a rigid heat conductor with microperiodic material structure, which describes the aforementioned effects on the time-dependent heat transfer processes. At the same time the range of physical applicability of the effective modulus theory will be outlined.

The approach to the proposed refined theory of heat conduction in composite materials is similar to that applied in a formulation of the refined macro-elastodynamics, WoźNIAK [4] being based on certain modelling hypotheses which will be given in Sec. 2. In Sec. 3, the governing relations of the refined heat conduction theory will be discussed, and in Sec. 4 we recall the effective modulus theory in the form given by MATYSIAK and WoźNIAK [3]. In order to compare these theories, in Sec. 5, the special problem is solved in the framework of both averaged models. The general conclusions are formulated in Sec. 6. The main result is that the proposed refined theory of heat conduction in micro-periodic composites has to be applied if we deal with nonstationary problems and that the effective modulus theories have the range of applicability rather restricted to the steady-state problems.

\section{List of symbols}

\begin{tabular}{|c|c|c|c|}
\hline$a, b, c, \ldots$ & superscripts run over $1,2, \ldots, n ; n \geqq 1$ & 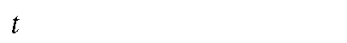 & time coordinate \\
\hline$c=c(x)$ & specific heat per unit volume in $\Omega \backslash \Gamma$ & $V \equiv\left(0, l_{1}\right) \times\left(0, l_{2}\right) \times\left(0, l_{3}\right)$ & representative volume element (r.v.e.) \\
\hline & $\begin{array}{l}\text { stands for a jump of an arbitrary func- } \\
\text { tion } F \text { across a surface } \Gamma\end{array}$ & $\boldsymbol{x} \equiv\left(x_{1}, x_{2}, x_{3}\right)$ & points belonging to $\bar{\Omega}$ \\
\hline$\langle f\rangle=\left(l_{1} l_{2} l_{3}\right)^{-1} \int_{V} f(x) \mathrm{d} v$ & $\begin{array}{l}\text { where } \mathrm{d} v \equiv \mathrm{d} x_{1} \mathrm{~d} x_{2} \mathrm{~d} x_{3} \\
\text { averaged value of a }\end{array}$ & Greek symbols & \\
\hline & $V$-periodic function $f(\cdot)$ & $\Phi^{a}=\Phi^{a}(\boldsymbol{x}, t)$ & temperature corrector fields \\
\hline $\begin{array}{l}h=h(\boldsymbol{x}, t) \\
h_{i}=h_{i}(\boldsymbol{x}, t)\end{array}$ & $\begin{array}{l}\text { heat supply across the unit area of } \partial \Omega \\
\text { heat flux vector in } \Omega\end{array}$ & $\Gamma$ & $\begin{array}{l}\text { interface between the adjacent constit- } \\
\text { uents in } \Omega\end{array}$ \\
\hline$i, j, k, l$ & $\begin{array}{l}\text { subscripts run over } 1,2,3 \text {, and are } \\
\text { related to the orthogonal cartesian } \\
\text { coordinates } x_{i} \text { parameterized } \\
\text { a region } \Omega\end{array}$ & $\begin{array}{l}\varrho=\varrho(x) \\
\Theta=\Theta(x, t) \\
\vartheta=\vartheta(x, t)\end{array}$ & $\begin{array}{l}\text { mass density in } \Omega \backslash \Gamma \\
\text { macro-temperature field } \\
\text { temperature field in a rigid } V \text {-periodic } \\
\text { conductor } \bar{\Omega}\end{array}$ \\
\hline$k_{i j}=k_{i j}(\boldsymbol{x})$ & heat conductivity tensor in $\Omega \backslash \Gamma$ & $\Omega$ & region occupied by a $V$-periodic rigid \\
\hline & unit normal vector to a surface $\Gamma$ or $\partial \Omega$ & & $\begin{array}{l}\text { conductor } \\
\text { region } \Omega \text { together with its boundary } \partial\end{array}$ \\
\hline $\begin{array}{l}q=q(\boldsymbol{x}, t) \\
s^{a}=s^{a}(\boldsymbol{x})\end{array}$ & $\begin{array}{l}\text { heat supply per unit mass in } \Omega \\
\text { micro-shape functions }\end{array}$ & $\Omega \backslash \Gamma$ & remaining part of $\Omega$ \\
\hline
\end{tabular}

\section{Modelling procedure}

The starting point of the modelling approach are the well known equations of the heat conduction in a rigid conductor. The energy balance yields the well known conditions $c \dot{\vartheta}-h_{i, i}=\varrho q$ in $\Omega \backslash \Gamma, h_{i} n_{i}=h$ on $\partial \Omega,\left[h_{i}\right] n_{i}=0$ on $\Gamma$, which can be jointly written down in the weak form

$$
\int_{\Omega} h_{i} \delta \vartheta, i \mathrm{~d} v=\oint_{\partial \Omega} h \delta \vartheta \mathrm{d} a+\int_{\Omega}(\varrho q-c \dot{\vartheta}) \delta \vartheta \mathrm{d} v,
$$

which has to hold for an arbitrary, sufficiently regular, test function $\delta \vartheta$. Eq. (1) has to be considered together with the Fourier law

$$
h_{i}(\boldsymbol{x}, y)=k_{i j}(\boldsymbol{x}) \vartheta_{, j}(\boldsymbol{x}, t), \quad \boldsymbol{x} \in \Omega \backslash \Gamma .
$$

Functions $k_{i j}(\cdot), c(\cdot), \underline{Q}(\cdot)$ are $V$-periodic and piecewise constant, attaining different constant values in pertinent constituents of the medium under consideration. Since in a composite body the length dimensions of the r.v.e. are very small compared to the minimum characteristic length dimension $L$ of $\Omega$, then $l \equiv \max \left\{l_{1}, l_{2}, l_{3}\right\}$, can be treated as a certain small parameter $l \ll L$, and Eqs. (1), (2) have the microperiodic highly oscillating coefficients. Hence, these equations cannot be directly applied to the computational analysis of engineering problems and constitute here only a starting point of the modelling procedure.

The procedure leading from Eqs. (1), (2) to the refined averaged model of the heat transfer problem will be based on certain heuristic hypotheses. In order to formulate these hypotheses we recall, following WoźNIAK [4], the auxiliary concept of the regular $V$-macro function, and that of the micro-shape functions.

Function $F(x, t)$ defined on $\Omega \times[0, \infty)$ is called $V$-macro function (related to a certain small macro-accuracy parameter $\lambda$ ) provided that for every $\boldsymbol{x}^{\prime}, \boldsymbol{x}^{\prime \prime} \in \Omega$, if $\boldsymbol{x}^{\prime}-\boldsymbol{x}^{\prime \prime} \in V$ then $\left|F\left(\boldsymbol{x}^{\prime}\right)-F\left(\boldsymbol{x}^{\prime \prime}\right)\right|<\lambda$. If the similar conditions also hold for all derivatives of $F$ (including time derivatives) then $F(\cdot)$ is called the regular $V$-macro function.

The sequence of $n$ linear independent continuous $V$-periodic function $s^{a}(\boldsymbol{x}), a=1, \ldots, n$, having piecewise continuous first derivatives, is referred to as the system of microshape functions if: $\left\langle s^{a}\right\rangle=0, s^{a}(x) \in 0(l)$ and the values of the first derivatives of $s^{a}$ are independent of the small parameter $l: s_{, i}^{a}(\boldsymbol{x}) \in 0(1)$. Moreover, the derivatives $s_{, i}^{a}$ can suffer jump 
discontinuities only on the interfaces between the adjacent constituents of the composite. The choice of the micro-shape functions is related to a certain microdiscretization of the r.v.e. and depends on the character of the problem under consideration. Roughly speaking, microshape functions play a role similar to that of the shape functions in the finite element method, but are restricted to the r.v.e. of a periodic composite structure.

Now, we formulate three basic hypotheses of the proposed modelling approach.

Temperature Distribution Hypothesis (TDH). The distribution of a temperature field in $V$-periodic composite will be assumed in the form

$$
\vartheta(\boldsymbol{x}, t)=\Theta(\boldsymbol{x}, t)+s^{a}(\boldsymbol{x}) \Phi^{a}(\boldsymbol{x}, t),
$$

where $\Theta(\cdot)$ and $\Phi^{a}(\cdot)$ are arbitrary regular $V$-macro functions and $s^{a}(\cdot)$ are postulated a priori micro-shape functions.

In the sequel functions $\Theta$ and $\Phi^{a}$ are treated as the new basic unknowns and will be referred to as the macro-temperature and the temperature correctors, respectively.

Energy Balance Assumption (EBA). The energy balance equation in its weak form (1) is assumed to be consistent with TDH and hence holds for every

$$
\delta \ni(x)=\delta \Theta(x)+s^{a}(x) \delta \Phi^{a}(x),
$$

where $\delta \Theta(\cdot)$ and $\delta \Phi^{a}(\cdot)$ are arbitrary regular $V$-macro functions.

Macro-Modelling Approximation (MMA). In calculations of the balance equation (1) by using Eqs. (2)-(4), terms $O(\lambda)$ are neglected in integrals over $\Omega$ and terms $0(l)$ are neglected in integrals over $\partial \Omega$.

Combining Eqs. (1) - (4) and using MMA, after rather lengthy manipulations, we arrive at the system of governing equations for the macro-temperature $\Theta$ and the temperature correctors $\Phi^{a}$. Setting aside all calculations we summarize the obtained results in the subsequent section.

\section{Refined theory (RT)}

The governing equations for $\Theta$ and $\Phi^{a}$ can be written down in the form of the following averaged balance equations:

$$
\begin{aligned}
& \langle c\rangle \dot{\Theta}(x, t)-H_{i, i}(x, t)=\langle\varrho q\rangle, \\
& \left\langle c s^{a} s^{b}\right\rangle \dot{\Phi}^{b}(x, t)+G^{a}(x, t)=0 ; \quad x \in \Omega, \quad t>0,
\end{aligned}
$$

where $H_{i}$ is the averaged heat flux and $G^{a}$ are called microheat fluxes, given by the macro-constitutive equations

$$
\begin{aligned}
& H_{i}=\left\langle k_{i j}\right\rangle \Theta_{, j}+\left\langle k_{i j} s_{, j}^{a}\right\rangle \Phi^{a}, \\
& G^{u}=\left\langle k_{i j} s_{, j}^{a}\right\rangle \Theta_{, i}+\left\langle k_{i j} s_{, i}^{a} s_{, j}^{b}\right\rangle \Phi^{b} .
\end{aligned}
$$

At the same time we obtain the averaged natural boundary conditions

$$
H_{i}(x, t) n_{i}(x)=h(x, t), \quad x \in \partial \Omega, \quad t>0 .
$$

Under extra denotation $C^{a b} \equiv\left\langle c s^{a} s^{b}\right\rangle l^{-2}$, from the aforementioned equations we obtain

$$
\begin{aligned}
& \langle c\rangle \dot{\Theta}(x, t)-\left\langle k_{i j}\right\rangle \Theta_{, i j}(x, t)-\left\langle k_{i j} j_{, j}^{a}\right\rangle \Phi_{, i}^{a}(x, t)=\langle\varrho q\rangle, \\
& l^{2} C^{a b} \dot{\Phi}^{b}(x, t)+\left\langle k_{i j} s_{, i}^{a} s_{, j}^{b}\right\rangle \Phi^{b}(x, t)+\left\langle k_{i j} s_{, j}^{a}\right\rangle \Theta_{, i}(x, t)=0 ; \quad x \in \Omega, \quad t>0 .
\end{aligned}
$$

Since $\left\langle c s^{a} s^{b}\right\rangle \in 0\left(l^{2}\right)$ and $s_{, i}^{a} \in 0(1)$, then $C^{a b} \in 0(1)$ and all averaged modulae in Eqs. (5) are independent of the microstructure length parameter $l$. This parameter occurs in the explicit form in the second from Eqs. (5). The obtained result represents the system of $n+1$ equations with constant coefficients for a macrotemperature $\Theta$ and $n$ temperature correctors $\Phi^{a}$. Due to the presence of the parameter $l$, this system describes the microstructure length-scale effect on the heat conduction. Such situation does not take place in effective modulus theories where all terms of an order $0(l)$ are neglected. That is why we shall refer the obtained model to as the refined (averaged) theory of heat conduction in a micro-periodic rigid conductor. The characteristic feature of this theory is that the temperature correctors $\Phi^{a}$ are governed by ordinary differential equations involving the first time derivatives of $\Phi$. Hence temperature correctors are certain internal balance variables, not depending on the boundary conditions. Hence Eqs. (5) have to be considered together with one boundary condition for $\Theta$, and $n+1$ initial conditions for $\Theta$ and $\Phi^{a}$. Let us also observe, that the solution to the initial-boundary value problem of the refined theory of heat conduction have a physical sense only if $\Theta, \Phi^{a}$ are regular $V$-macro functions. Hence, also $H_{i}$ and $G^{a}$ are $V$-macro functions. This fact imposes certain restriction on the distribution of heat supply $h$ across the boundary. 


\section{Effective modulus theory (EMT)}

The effective modulus theory of heat conduction can be based on TDH and EBA, and on the limit passage $l \rightarrow 0$ under the condition that the ratios $l_{i} / l$ of the r.v.e. are constant. This procedure leads to the equation with constant coefficients for the macro-temperature $\Theta$

$$
\langle c\rangle \dot{\Theta}(x, t)-K_{i j} \Theta_{, i j}(x, t)=\langle\varrho q\rangle ; \quad x \in \Omega, \quad t>0,
$$

where the tensor with the components $K_{i j}$, defined by

$$
K_{i j}=\left\langle k_{i j}\right\rangle-\left\langle k_{i k} s_{, k}^{a}\right\rangle H^{a b}\left\langle k_{j l} s_{, l}^{b}\right\rangle,
$$

where $H^{a b}\left\langle k_{i j} s_{i, i}^{b} s_{j}^{c}\right\rangle=\delta^{a c}$, is called the effective thermal conductivity tensor. Let us observe, that EMT can be obtained directly from RT by neglecting the first term in Eq. (5) $)_{2}$, which makes it possible to eliminate $\Phi^{a}$ from Eqs. (5). The discussion and applications of Eq. (6) can be found in the paper by MATYSIAK and WoźNIAK [3]. In this contribution we recall the effective modulus theory only in order to compare RT and EMT, which will be done in the subsequent section.

\section{Example}

The aim of this example is to compare two solutions to a certain simple heat conduction problem, which will be obtained in the framework of RT and EMT. To formulate this problem we consider a thick laminated two-component periodic layer, bounded by the coordinate planes $x_{1}= \pm L$. The representative element of this layer is made of two laminae of thicknesses $l^{\prime}$, $l^{\prime \prime}$, where $l=l^{\prime}+l^{\prime \prime}$ is sufficiently small compared to $L, l \ll L$ (cf. Fig. 2). We introduce one micro-shape function $s\left(x_{1}\right)$, given in $[0, l]$ by $s(0)=s(l)=-l / 2, s\left(l^{\prime}\right)=l / 2$ and linear in $\left[0, l^{\prime}\right]$ and $\left[l^{\prime}, l\right]$. Moreover, we assume that the heat conduction across the layer is independent of coordinates $x_{2}, x_{3}$. Hence Eq. (3) has the form

$$
\vartheta\left(x_{1}, t\right)=\Theta\left(x_{1}, t\right)+s\left(x_{1}\right) \Phi\left(x_{1}, t\right),
$$

and we deal only with one temperature corrector $\Phi$. Setting $k \equiv k_{11}, \delta^{2}=l^{2} / 12$, and neglecting the heat supply $q$, from Eqs. (5) of RT we obtain

$$
\begin{aligned}
& \langle c\rangle \dot{\Theta}\left(x_{1}, t\right)-\langle k\rangle \Theta_{, 11}\left(x_{1}, t\right)-\left\langle k s_{, 1}\right\rangle \Phi_{, 1}\left(x_{1}, t\right)=0, \\
& \delta^{2}\langle c\rangle \dot{\Phi}\left(x_{1}, t\right)+\left\langle k\left(s_{, 1}\right)^{2}\right\rangle \Phi\left(x_{1}, t\right)+\left\langle k s_{, 1}\right\rangle \Theta_{, 1}\left(x_{1}, t\right)=0 ; \quad x_{1} \in(-L, L), \quad t>0 .
\end{aligned}
$$

Let the boundary condition be given by $\Theta(L, t)=\Theta(-L, t)=\Theta_{L}$ where $\Theta_{L}=$ const. For the sake of simplicity let the initial condition for the macro-temperature be assumed in the form $\Theta\left(x_{1}, 0\right)=\Theta_{L}+\Theta_{0} \cos \left(\pi x_{1} / 2 L\right), x_{1} \in(-L, L)$, $\Theta_{0}$ being constant. For the temperature corrector $\Phi$ we assume the trivial initial condition $\Phi\left(x_{1}, 0\right)=0, x_{1} \in(-L, L)$;
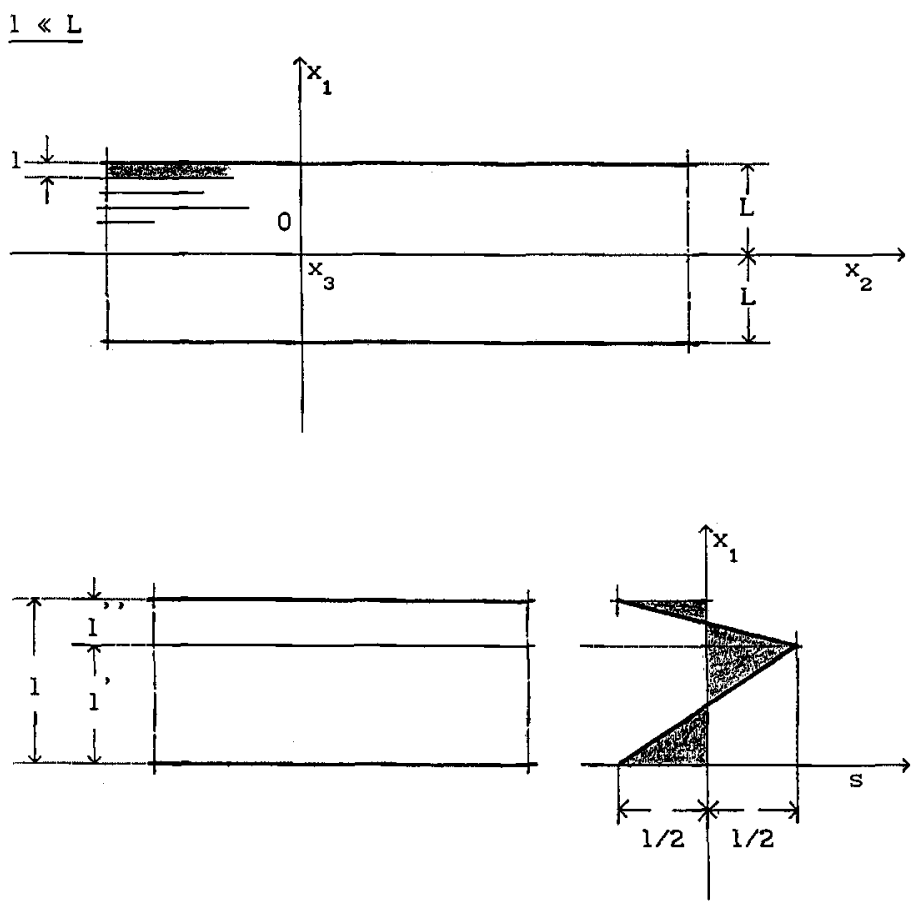

Fig. 2. Scheme of a laminated layer and a diagram of a micro-shape function $s(\cdot)$ in the interval $[0, l]$ 
it means that there is no micro-disturbances in the initial distribution of temperature. The solution to this problem can be written down in the form

$$
\begin{aligned}
& \Theta\left(x_{1}, t\right)=\Theta_{L}+\Theta_{0} \exp \left(-\lambda_{1} t\right) \cos \left(\pi x_{1} / 2 L\right)+0\left(\delta^{2}\right), \\
& \Phi\left(x_{1}, t\right)=\Theta_{0}\left\langle k s_{, 1}\right\rangle\left\langle k\left(s_{, 1}\right)^{2}\right\rangle^{-1}(\pi / 2 L)\left[\exp \left(-\lambda_{1} t\right)-\exp \left(-\lambda_{2} t\right)\right] \sin \left(\pi x_{1} / 2 L\right)+0\left(\delta^{2}\right),
\end{aligned}
$$

where

$$
\begin{aligned}
& i_{1}=K\langle c\rangle^{-1}(\pi / 2 L)^{2}-K\left\langle k s_{, 1}\right\rangle^{2}\langle c\rangle^{-1}\left\langle k\left(s_{, 1}\right)^{2}\right\rangle^{-1}(\pi / 2 L)^{4} \delta^{2}+0\left(\delta^{4}\right), \\
& K \equiv\langle k\rangle-\left\langle k s_{, 1}\right\rangle^{2}\left\langle k\left(s_{, 1}\right)^{2}\right\rangle^{-1}, \\
& i_{2}=\left\langle k\left(s_{, 1}\right)^{2}\right\rangle\langle c\rangle^{-1} \delta^{-2}+\left\langle k s_{, 1}\right\rangle^{2}\langle c\rangle^{-1}\left\langle k\left(s_{, 1}\right)^{2}\right\rangle^{-1}(\pi / 2 L)^{2}+0\left(\delta^{2}\right) .
\end{aligned}
$$

For the averaged heat flux $H_{1}=\langle k\rangle \Theta_{, 1}+\left\langle k s_{, 1}\right\rangle \Phi$ we obtain the formula

$$
H_{1}=-\Theta_{0} \pi / 2 L\left[K \exp \left(-\hat{\lambda}_{1} t\right)+\left\langle k s_{, 1}\right\rangle^{2}\left\langle k\left(s_{, 1}\right)^{2}\right\rangle^{-1} \exp \left(-\hat{\lambda}_{2} t\right)\right] \sin \left(\pi x_{1} / 2 L\right),
$$

and the oscillations $\Delta \vartheta_{, 1}$ of temperature gradients between every two adjacent laminae, given by $\Delta \vartheta_{.1} \equiv \Phi\left(x_{1}, t\right) l^{2} / l^{\prime \prime}$ (where $x_{1}$ is related to the interface between laminae) are

$$
\Delta \gamma_{, 1}=\Theta_{0}\left\langle k s_{, 1}\right\rangle\left\langle k\left(s_{, 1}\right)^{2}\right\rangle^{-1}\left(\pi l^{2} / 2 L l^{\prime} l^{\prime \prime}\right)\left[\exp \left(-\lambda_{1} t\right)-\exp \left(-\lambda_{2} t\right)\right] \sin \left(\pi x_{1} / 2 L\right)+0\left(\delta^{2}\right) .
$$

Eqs. (8) - (10) hold for every $x_{1} \in[-L, L]$ and $t \geqq 0$.

Equations of the effective modulus theory can be obtained from Eqs. (7) by neglecting the underlined term involving the parameter $\delta^{2}=1^{2} / 12$. In this case the temperature corrector $\Phi\left(x_{1}, t\right)$ is governed by the linear algebraic equation and
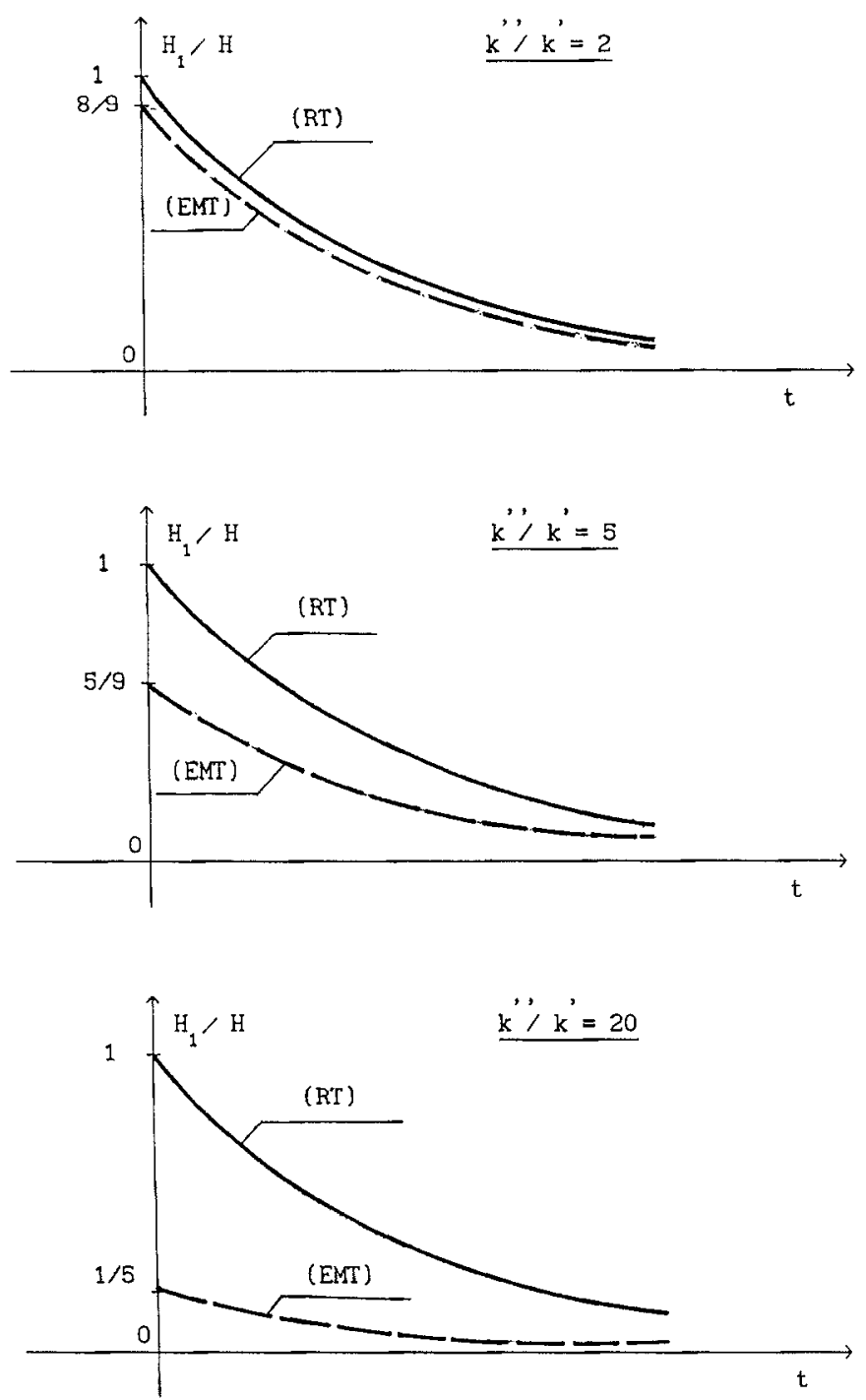

Fig. 3. Diagram of a heat flux $H_{1}$ for $x_{1}=-L ; k^{\prime \prime} / k^{\prime}$ is taken as a parameter, and $H=\pi\langle k\rangle \Theta_{0} / 2 L$ 
the initial condition $\Phi\left(x_{1}, 0\right)=0$ may not hold. The solution to the problem considered in the framework of EMT is

$$
\begin{aligned}
& \Theta\left(x_{1}, t\right)=\Theta_{0} \exp (-\lambda t) \cos \left(\pi x_{1} / 2 L\right)+\Theta_{L}, \\
& \Phi\left(x_{1}, t\right)=\Theta_{0}\left\langle k s_{, 1}\right\rangle\left\langle k\left(s_{, 1}\right)^{2}\right\rangle^{-1}(\pi / 2 L) \exp (-\lambda t) \sin \left(\pi x_{1} / 2 L\right),
\end{aligned}
$$

where

$$
\lambda=K\langle c\rangle^{-1}(\pi / 2 L)^{2} .
$$

The averaged heat flux $H_{1}$ is equal to

$$
H_{1}=-\Theta_{0} K \pi / 2 L \exp (-\lambda t) \sin \left(\pi x_{1} / 2 L\right),
$$

and the oscillations of temperature gradients between adjacent laminae are

$$
\Delta \vartheta_{, 1}=\Theta_{0}\left\langle k s_{, 1}\right\rangle\left\langle k\left(s_{, 1}\right)^{2}\right\rangle^{-1}\left(\pi l^{2} / 2 L l^{\prime} l^{\prime \prime}\right) \exp (-\lambda t) \sin \left(\pi x_{1} / 2 L\right) .
$$

Eqs. (11) - (13) hold for every $x_{1} \in[-L, L]$ and $t \geqq 0$.

Comparing formulae (8) $-(10)$ related to RT, and formulae (11)-(13) obtained in the framework of EMT it can be seen that the distribution of the macro-temperature obtained from EMT can be treated as a certain approximation (of an order $0\left(\delta^{2}\right)$ ) of that obtained from RT. However, the results obtained in the framework of EMT for the averaged heat flux $H_{1}$ and the temperature oscillation $\Delta \vartheta, 1$ are uncorrect. In Fig. 3, there are shown diagrams of a heat flux for $x_{1}=-L$, where the ratio of heat conductivities $k^{\prime}, k^{\prime \prime}$ of adjacent laminae is taken as a parameter, $H \equiv \pi \Theta_{0}\langle k\rangle / 2 L$, and $l^{\prime}=l^{\prime \prime}$.

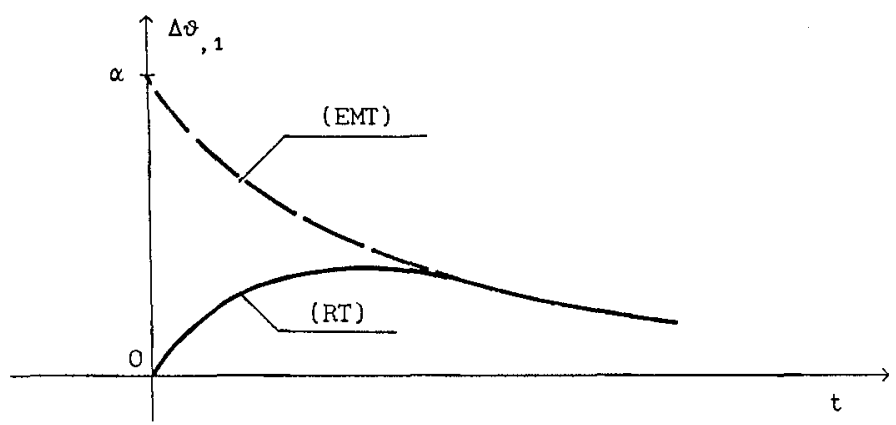

Fig. 4. A diagram of oscillations of temperature gradients between adjacent laminae for $x_{1}=L ; \alpha=2 \pi\left\langle k s_{, 1}\right\rangle \Theta_{0} / L\left\langle k\left(s_{.1}\right)^{2}\right\rangle$

In Fig. 4, the oscillations of temperature gradients between adjacent laminae are shown for $x_{1}=L$; here also $l^{\prime}=l^{\prime \prime}$ and $\alpha \equiv 2 \pi \Theta_{0}\left\langle k s_{, 1}\right\rangle / L\left\langle k\left(s_{, 1}\right)^{2}\right\rangle$. The discrepancies between RT and EMT hold only for small times, for which values of $\exp \left(-\lambda_{2} t\right)$ are not neglectibly small compared to those of $\exp \left(-\lambda_{1} t\right)$. Let us also observe that for "weak" inhomogeneities, where the ratio $k^{\prime} / k^{\prime \prime}$ is approximatively equal to 1, results obtained from EMT can be treated as approximations of those of RT.

\section{Conclusions}

The investigations of governing equations for RT and EMT as well as the results obtained in Sec. 5 lead to the following general conclusions:

(i) In stationary problems RT and EMT coincide.

(ii) For homogeneous media RT reduces to the well known formulations of the heat conduction problems provided that the trivial initial conditions for all temperature correctors are prescribed.

(iii) In nonstationary problems the microstructure length-scale effects on the heat conduction cannot be neglected and hence in these problems RT has to used as the tool of analysis.

Moreover, for some special nonstationary problems, the macrotemperature field calculated on the basis of EMT can represent a good approximation of this field obtained in the framework of RT. Such situation does not occur, as a rule, if we deal with calculations of heat fluxes.

More general approach to the heat conduction problems in micro-periodic materials, in which the deformation of a conductor is taken into account, will be presented in a separate paper. 


\section{References}

1 Bensoussan, A.; Lions, J. L; Papanicolaou, G.: Asymptotic analysis of periodic structures. North-Holland, Amsterdam 1980.

2 Bakinvalov, N. S.: Panasenko, G. P.: Osrednienie processov v periodicheskikh sredakh [in Russian]. Nauka, Moskva 1984.

3 Matysiak, S.; Woźniak, CZ.: Micromorphic effects in a modelling of periodic multilayered elastic composites. Int. J. Eng. Sci. 25 (1987) 5, 549-559.

4 Woźniak. Cz.: Refined macrodynamics of periodic structures. Arch. Mech. 45 (1993) 3, $295-304$.

Received February 16, 1995, revised and accepted May 19, 1995

Addresses: Prof. Dr. Habil.-Eng. CzeSeaw Woźniak, Doc. Dr. Habil.-Eng. Zbigniew F. BaCzyński (to whom correspondence should be addressed), Polish Academy of Sciences, Institute of Fundamental Technological Research, Center of Mechanics, Świętokrzyska 21, PL-00-049 Warszawa, Poland; Prof. Dr. Habil.-Eng. Margaret WoźniaK, Technical University Lódź, Department of Geotechnical and Structure Engineering, Al. Politechniki 6, PL-93-950 Łódż, Poland 